\title{
Michel Cartry et la question de l'espace-corps
}

\section{Danouta Liberski-Bagnoud}

\section{(2) OpenEdition}

\section{Journals}

Édition électronique

URL : http://journals.openedition.org/span/1696

DOI : $10.4000 /$ span. 1696

ISSN : 2268-1558

\section{Éditeur}

École pratique des hautes études. Sciences humaines

\section{Édition imprimée}

Date de publication : 1 janvier 2014

Pagination : 79-100

ISBN : 9782909036434

ISSN : 0294-7080

\section{Référence électronique}

Danouta Liberski-Bagnoud, «Michel Cartry et la question de l'espace-corps », Systèmes de pensée en Afrique noire [En ligne], 19 | 2014, mis en ligne le 05 février 2020, consulté le 11 février 2020. URL http://journals.openedition.org/span/1696 ; DOI : 10.4000/span.1696 


\section{Michel Cartry et la question de l'espace-corps}

\section{Danouta Liberski-Bagnoud}

Directrice de recherche au CNRS Institut des mondes africains (IMAF) Institut d'études avancées de Nantes

En 1973, alors qu'il se présentait à l'École Pratique des Hautes Études, Michel Cartry avait indiqué que son projet d'enseignement porterait sur l'analyse des systèmes de pensée en Afrique noire, et qu'il conduirait cette analyse en suivant deux directions de recherche, l'une sur l'idéal du corps unifié et le thème du corps morcelé à travers les mythes, les contes et les pratiques rituelles, l'autre sur les systèmes divinatoires. Assurément, il aura développé ce programme jusqu'en ses moindres plis, démontrant, chemin faisant, à quel point ces deux "thématiques ", du corps et de la parole divinatoire, qui peuvent apparaître de prime abord disjointes, sont en réalité solidaires et renouvellent l'abord des recherches sur la nature et la fonction du rituel. La recherche qu'il proposait d'entreprendre sur l'image du corps se démarquait d'un énième inventaire raisonné " des composantes de la personne ", très en vogue à l'époque de la rédaction du projet'. L'auteur entendait centrer l'enquête sur le thème du corps morcelé, redoublé ou multiplié, tel qu'on le trouve traité au plan des rites, en particulier les rites qui entourent les naissances exceptionnelles, gémellaires ou péri gémellaires, comme au plan du dire, à l'exemple de ces contes gourmantché qui mettent en scène « la femme et ses 444 bouches ", "les 333 enfants du chef fondu en un ", "la bouche prononçant l'oraison funèbre des autres organes du corps ", ou à celui des grands récits mythiques ouest africains qui décrivent l'idéal d'un corps unifié sous la forme

\footnotetext{
${ }^{1}$ Le colloque sur la notion de personne qui s'est tenu en 1971 et, auquel il a participé (il en fut même l'une des chevilles ouvrières), faisait encore la part belle aux enquêtes sur les représen. tations, notions, croyances relatives aux composantes du corps et « spirituelles ».
} 
${ }^{2}$ La seconde année de son enseignement a plus spécifiquement porté sur l'analyse des catégories de pensées liées aux idées de procréation et de génération, dans le cadre d'une théorie du destin. Cette thématique s'inscrivait dans le prolongement des recherches menées l'année précédente sur l'image qu'une société donnée se fait du corps des jumeaux à travers ses conceptions génétiques et embry. ologiques, implicites et explicites. (Cartry 1973 \& 1974). de couples de jumeaux de sexe opposé, présentés comme réalisant une sorte de perfection ontologique. L'enjeu était de repérer en chacune des aires culturelles examinées le ou les modèles idéaux spécifiques de corps unifié (en prenant en compte les modèles très différents apportés par l'Islam), avec ce que de tels modèles impliquent d'une conception originale des rapports des parties au tout, et d'en définir les propriétés. De manière explicite, ce volet de son enseignement s'inscrivait dans une réflexion philosophique et historique plus large sur les systèmes de représentations du corps dans les sociétés qui se sont développées hors du machinisme industriel. À l'horizon de cette recherche particulière, se tient en effet la question de savoir s'il existe des traits communs aux "images collectives du corps " qu'ont élaborés dans le temps et l'espace des sociétés où le corps humain est resté " le mesurant des choses ", où la production dépend directement du travail du corps de chacun, de sa force et de son habileté. Après deux années consacrées à la mise en place des jalons d'une recherche portant sur les croyances, les mythes et les rites liés aux jumeaux ${ }^{2}$, Michel Cartry ouvrit une enquête de longue haleine sur la catégorie de l'espace. Il avait changé d'angle d'attaque, mais non d'objet.

Pourquoi ce déplacement? Par quel cheminement de la pensée a-t-il été conduit à aborder l'image du corps, que partagent les membres d'une même collectivité, par le biais d'une interrogation sur le rapport du corps à l'espace et au territoire que construisent ces mêmes collectivités? La notion " d'espace-corps » qu'il propose pour la première fois dans un écrit de 1979 livre les éléments de la réponse. C'est l'élaboration de cette notion en un véritable concept dans l'œuvre de Michel Cartry qui est l'objet de cette communication.

On ne lit pas de la même manière une ouvre en train de se faire, dont on prend connaissance dans le temps même où elle se conçoit, et les écrits figés avec lesquels la disparition de leur auteur nous laisse en tête à tête. C'est une évidence, presque une banalité, mais qui, dans le cas qui nous occupe, se trouve d'autant plus exact que Michel 
Cartry avait une conception toute socratique de son enseignement. Il ne cherchait jamais à imposer ses idées, il ne délivrait aucune doctrine. Jamais pédant ou pontifiant, il accordait une écoute attentive et exigeante à ce que lui rapportaient ses élèves, qu'il s'agisse de leur expérience de terrain ou de leurs lectures. Loin de toute position d'autorité, il marquait son enthousiasme par un feu roulant de questions auquel il soumettait son interlocuteur, non pas depuis un supposé savoir dont il aurait été le maître, mais tel un condisciple qui cherche avec vous ce que tout cela pourrait bien vouloir dire. Passeur plutôt que berger d'un troupeau de disciples, il indiquait des voies d'interprétations possibles, des impasses théoriques déjà éprouvées par lui, mais il vous laissait toujours maitre d'essayer une toute autre voie. En sa présence, on était de la sorte conduit à ne retenir de son questionnement théorique que ce qui venait éclairer, mettre en ordre, trouver sens à nos propres matériaux d'enquête. On se laissait porter par l'idée qu'il était seul à détenir la clef de compréhension de la trajectoire intellectuelle qu'il était en train de suivre. Maintenant qu'il n'est plus là pour la prolonger, un retour à ses écrits s'impose ; l'œuvre se lisant désormais comme un objet clos, on peut tenter d'en repérer les lignes de cohérence. La notion d'espace-corps cristallise l'une de ces lignes de cohérence, et leste de son poids conceptuel certaines des questions majeures autour desquelles sa recherche sur la nature et la fonction du rituel s'est nouée.

Relevons d'emblée l'apparent paradoxe : cette notion qui a orienté un grand nombre de ses enquêtes thématiques n'apparaît sous sa plume qu'à deux reprises, en deux textes publiés à presque trente ans de distance (Cartry 1979, 2005). Dans son enseignement, cependant, il y fera référence à plusieurs reprises, alors qu'il s'interrogeait sur les propriétés singulières de ces espèces d'espaces que sont les différents lieux de la maison dans ces temps où ils sont investis par le rite, un sanctuaire envahi par la souillure suite à la rupture d'un interdit fondamental, ou encore cette construction éphémère en brousse qui est appelée, dans la littérature ethnologique, un "camp de circoncision $»^{3}$. Dans les pages non

${ }^{3}$ Cartry 1984, 1989, 1990, 1991. 
${ }^{4}$ Un premier dépouil. lement des archives confiées à la Biblio. thèque Julien Gracq de I'Institut d'Etudes avancée de Nantes a mis au jour, dans un dossier divination, deux plans d'ouvrages. Le premier, le plus ancien, intitulé

" La bouche de la terre. Essai sur la divination et le rituel en pays gourmantché " comprend une dizaine de pages où, de façon synthétique, sont notés ses " axiomes, définitions, postulats sur le rituel », un plan général et les linéaments d'une introduction. Le second, intitulé « La bouche de l'oracle. Questionner et dire l'oracle dans une forme africaine d'idiome géoman. tique », comporte une introduction substanti. elle d'une trentaine de pages où sont dıscutés les thèses classiques sur la divination. Ces pages témoignent de l'ampleur du projet que la mort a laissé Inachevé. La citation donnée en fin de texte provient du premier document. publiées d'un projet d'ouvrage ${ }^{4}$, Michel Cartry donne un nouveau tour à la notion, en l'insérant dans une réflexion sur le corps, le langage et le rite. Ce document renforce l'hypothèse selon laquelle cette notion est l'un des points de capiton de l'ensemble de sa recherche. Transformant le regard et l'écoute dans l'abord de tout un ensemble de phénomènes ou d'événements que rien en apparence ne permettrait de lier a priori, "l'espace-corps " fonctionne tel un concept.

Je ne chercherai pas ici à retracer en détail le cheminement d'une pensée qui, parti de l'analyse des croyances et les représentations concernant les jumeaux en Afrique (programme et première année d'enseignement) pour tenter de cerner l'image collective du corps propre que construit une société donnée, a pris la voie d'une enquête sur la structuration de l'espace vécu (à partir de la Conférence de la troisième année d'enseignement 75-76). Il suffit d'en rappeler le point de départ et quelques-uns des moments clefs. Au départ, assurément, il y eût la rencontre avec les raisonnements complexes que tiennent les Dogon à propos de la notion de gémellité, tels qu'ils affleurent dans ce registre spécifique de la parole qu'est le dire mythologique auquel ont recours les responsables rituels au cours d'une performance rituelle ou lorsqu'ils entreprennent d'expliciter tel ou tel aspect de leur pratique religieuse (signes, monuments, objets, gestes, paroles). L'article écrit en collaboration avec Alfred Adler, "La transgression et sa dérision " (1971), témoigne de l'importance du rôle joué par cette rencontre, qui s'est faite à travers les écrits de Marcel Griaule et de Germaine Dieterlen. À première vue, la problématique abordée en ce texte n'a aucun lien immédiat avec un questionnement sur l'image collective du corps. Les auteurs entendent soumettre à un examen nouveau le problème des origines dans le champ de la parenté, problématique qui se ramène dans la littérature anthropologique à une interrogation sur la question de l'interdit de l'inceste. Sans chercher à récuser la validité de cette interrogation, les auteurs disent vouloir emprunter une direction différente, celle qu'indiquent les mythologies et qui a trait à ce qu'ils nomment 
un " en deçà de l'inceste "(Adler \& Cartry 1971 : 6). "En deçà ", car le mythe relate l'émergence d'un ordre, et qu'il ne saurait donc mettre en scène des personnages investis de rôles parentaux qui appartiennent à l'ordre constitué par la prohibition de l'inceste, en aval du mythe. En deçà, également, car la plupart des grandes cosmogonies, et singulièrement celle qui pénètre le système rituel des Dogon, ne parlent pas de personnes, mais d'entités "pré personnelles ", de créatures inachevées, d'avatars de membres disjoints, d'organes projetés dans l'espace, de morceaux d'un placenta cosmique duquel la terre sera faite. Centrant leur attention sur ce thème de l'unité et du morcellement de la création, les auteurs annoncent leur intention de suivre les avatars de la notion de gémellité dans la mythologie dogon, afin de s'aider des catégories et schèmes offerts par le mythe pour saisir la logique symbolique interne qui organise l'institution avunculaire, et, au-delà, l'ordre de la parenté qui la fonde. Ce n'est qu'au terme de cette étude extrêmement riche et approfondie, qui ouvre des voies originales dans l'abord de plusieurs questions récurrentes de la discipline, qu'est énoncé un autre enjeu théorique de cette enquête sur la notion de gémellité. Cet enjeu, les auteurs l'articulent sans détour : le problème central que pose la gémellité dans le récit mythique, annoncent-ils, est celui de "la fonction remplie par l'image du corps dans l'organisation dynamique des rapports de la société dogon avec elle-même et avec le monde "(Adler \& Cartry, 1971 : 43). La problématique, assurément, ne relève pas d'une anthropologie classique. Une brève mention faite aux travaux du Dr Gisela Pankow indique aux lecteurs que la notion d'"image du corps " dont il est ici question appartient au champ conceptuel de la psychanalyse où elle a reçu des élaborations théoriques successives. La notion est complexe, car elle ne désigne pas simplement une image. Synthèse dynamique des expériences émotionnelles d'une personne depuis sa venue au monde ${ }^{5}, l^{\prime}$ image du corps » doit être distinguée de l'image spéculaire (le corps tel qu'il est perçu par l'autre), mais aussi
${ }^{5}$ Selon certains psychanalystes, elle intègrerait les expériences sensori. elles depuis le temps prénatal de la vie intra.utérine.

(Françoise Doito, 1984). 
${ }^{6}$ Le philosophe

Osamu Nishitani donne un magnifique exemple de l'existence, dans la langue japonaise, d'au moins " deux » corps : celui anatomique de la médecine et celui que I'on habite au quotidien. (Nishitani, in Legendre, 2013: 298). des notions neurologiques telles que le schéma corporel, le corps ressenti, la proprioception etc., qui en font partie sans se confondre avec elle (Poupart 2014). Quelle que soit leur approche théorique, les psychanalystes mettent tous l'accent sur le pouvoir symbolisant de cette image mentale et synthétique du corps. Elle donne forme et contenu à cet inextricable dont parle Merleau-Ponty lorsqu'il évoque cet " ordre de phénomènes où nous sommes mêlés au monde et aux autres dans une confusion inextricable " (MerleauPonty 1945 : 518). Ce n'est pas l'anatomie comme référence au réel qui est ici en cause, la notion d' "image du corps" se référant uniquement à la façon dont un sujet, en fonction de son histoire personnelle "vit " son corps, l'habite ou peine à l'habiter. Il faut y insister, car cet abord n'est pas familier à l'anthropologie qui, le plus souvent, traite la référence au corps dans les mythes et les rites comme une référence parmi d'autres (il y aurait un code anatomique, physiologique comme il y a un code astronomique, culinaire, etc.), comme si le corps était une donnée immédiate de l'expérience humaine, comme s'il n'était pas toujours déjà pris dans du langage ${ }^{6}$. G. Pankow distingue deux fonctions symbolisantes de l'image du corps : la première se réfère au corps comme forme (Gestalt), qui s'offre comme modèle d'une structure spatiale dynamique, porteuse de lois relatives à la dialectique du tout à ses parties, du dedans au dehors, du contenant au contenu. La seconde fonction ne concerne plus la structure comme forme mais comme contenu et sens (Pankow 1977 : 19-20). Seule la première de ces fonctions retiendra l'attention des deux ethnologues, car elle leur semble ouvrir une voie féconde pour aborder autrement les spéculations dogon autour de corps redoublés de créatures gémellaires cosmiques. Depuis leur apparition comme ébauche de la différence dans l'œuf clos originel, les aventures de ces créatures et de leurs avatars président à l'émergence d'un monde qui, par le fait de la démesure de l'une d'entre elles, s'ouvre au temps et à l'espace où vont pouvoir prendre place et se déployer la succession des générations d'humains et la différenciation des segments de la société dogon. Sans revenir sur le détail de 
cette épopée fantastique, on retiendra, avec les auteurs, que le monde ainsi mis en place par le mythe " est un corps" non pas corps humain, mais corps de l'une de ces créatures célestes, sacrifiée et démembrée à la suite du désordre introduit par son jumeau dans le projet initial de création prévu par Amma (principe créateur, identifié à un Dieu suprême qui serait à la fois hors et dans sa création). Toute chose, catégorie, lignées, segments, villages, castes sont conçues comme le produit du partage, et l'image, des membres disjoints de cette créature sacrifiée. La terre elle-même est tirée de ce corps puisqu'elle est faite d'un morceau de son placenta céleste. Ce corps monde, qui s'offre à fois comme dedans, comme surface d'inscription et comme substance dont est tiré l'existant, il ne faut pas se précipiter, nous disent les auteurs, à n'y voir qu'une application particulière d'une représentation anthropomorphique de l'univers, selon un processus projectif ou un processus de liaison analogique. Un tel schéma serait en réalité réducteur, car il suppose implicitement l'existence d'une « coupure entre société et nature, entre individu et société, que la pensée et l'organisation sociale dogon démentent à tous les niveaux " (Adler $\&$ Cartry 1971 : 44). Ce qui se joue dans la dynamique de la recollection des parties dans le tout de ce corps céleste démembré puis reconstitué, comme dans le geste d'attribuer à toute chose, à tout être, une position sur ce corps monde, ce n'est rien moins que la condition de possibilité de leur discernabilité (ibidem). À cette reconstitution dans l'espace correspond également une reconstitution dans le temps, chaque moment du cycle de vie d'une personne étant identifié avec l'une des parties du corps de la créature céleste (sa tête, sa poitrine, ses bras, et finalement sa forme adulte d'un couple de jumeaux mixtes). Sans cesse menacé dans son intégrité par les désordres du vivant, ce corps monde doit continument être comme régénéré, rétabli dans sa totalité, par les sacrifices saisonniers. Cette recollection dans le temps et l'espace d'une "image du corps" portée tant par le mythe que le rituel permet à chacun d'appréhender et de penser les rapports dynamiques des parties (segment, lignage, maisonnée; étapes de la vie) entre elles et 
des parties au tout (le groupe, la trajectoire d'une vie singulière). Ainsi donc, avancent Adler et Cartry, " tout se passe comme si la fonction formelle de l'image du corps, [...] loin d'en laisser à chacun le soin de l'élaborer, était prise en charge par la société dogon, qui offre de la sorte à chacun de ses membres une surface de repérage où il puisse s'anticiper " (Adler \& Cartry 1971 : 46).

Ce texte témoigne de la fécondité qu'il y a à faire fonctionner dans le champ de l'interprétation anthropologique le concept psychanalytique d'image du corps, qui permet de penser en terme de co-construction l'élaboration de cette "image du corps " et celle de la structuration du monde extérieur. La question de savoir comment et pourquoi Michel Cartry est passé d'un projet de recherche sur l'image collective du corps à une enquête sur la structuration de l'espace vécu, tombe d'elle même. Image du corps et structure spatiale sont les deux faces d'une même question. Dans ses écrits ultérieurs, Michel Cartry n'aura plus explicitement recours à ce concept, mais il serait aisé de montrer qu'en toutes ses recherches liées à des questions d'espace et de territoire, la référence à l'image du corps y est constamment présente. La notion proprement anthropologique d'espace-corps qu'il propose, ne vient pas en lieu et place du concept psychanalytique d'image du corps, mais s'étaye sur elle et lui répond, en déplaçant l'accent sur des modalités spécifiques de construction de l'espace vécu dans les rites et les discours d'une communauté villageoise.

Pour en juger, il faut rappeler de quelle manière, et à quel moment de la réflexion, la notion d'espace-corps intervient pour la première fois dans l'article de 1979, "Du village à la brousse ou le retour de la question ". Le texte, écrit pour un ouvrage collectif qui rend hommage aux avancées théoriques de Lévi-Strauss sur le primat de la fonction symbolique, s'ouvre sur le constat que pour rendre compte du rapport qu'entretiennent les sociétés subsahariennes avec la brousse, la notion d'espace projectif (au sens établi par Piaget d'une représentation de l'espace du point de vue d'autrui) est insuffisante. En ces sociétés, avance d'emblée l'auteur, il a été maintes fois établi que l'espace 
de brousse est construit comme l'" autre du village ", vers lequel est systématiquement renvoyé tout ce qui survient au village d'anormal et d'étrange. Reformulant le constat en les termes de son ethnographie, Michel Cartry se propose de tester l'hypothèse suivante : l'espace de brousse fait fonction de référence «qui s'introduit comme tiers entre "l'être du village " et sa question, chaque fois que cette question surgit comme une énigme "(Cartry 1979 : 266). La brousse, ajoute-t-il, n'est pas pour autant le « lieu de la réponse, mais elle se présente comme une sorte d'opérateur logique autorisant une certaine permutation des termes dans la structure même de l'énigme "(ibidem). Ainsi posé l'enjeu du voyage, le lecteur est conduit " à prendre connaissance de l'environnement géographique où prennent place les rites et les activités liées à la brousse " dont il sera question dans le texte. Mais ce qui suit est bien autre chose qu'une simple description géographique? Par le biais d'une analyse serrée de faits de langue, de gestes du quotidien et de rites, l'auteur va cerner précisément ce qui, pour les Gourmantché, différencie fondamentalement le territoire du village où sont bâtis les maisons de l'espace ${ }^{8}$ de brousse où seuls les chasseurs sont conduits à séjourner plus ou moins longuement. La façon de penser cette différence, on pouvait sans douter, est très éloignée de celle qu'est amenée à construire le regard positiviste à partir du mode d'occupation des sols, de leurs usages différenciés, ou des ressources matérielles que ces deux catégories de terres offrent à l'exploitation des hommes. Il ne suffit pas, en effet, de décrire l'espace habité comme "anti-brousse ", ni la brousse comme un espace non humanisé, non domestiqué, non travaillé de la main de l'homme, pour comprendre la façon dont les Gourmantché pensent le rapport différencié qu'ils entretiennent vis-à-vis de ces deux catégories spatiales (Cartry 1975 : 88). Ce qui, pour eux, singularise la brousse du territoire villageois est tout d'abord l'absence de limites fixes, le " très lointain de la brousse " pouvant, selon les heures de la journée ou selon les événements, se trouver tout à coup, " très proche ", envahissant le village jusqu'aux clôtures de l'habitation, ou formant des enclaves dans le territoire du village. Une autre de ses caractéristiques est l'indistinction, l'absence de contours
${ }^{7}$ Elle s'appuie sur une recherche antérieure, longue et minutieuse, menée dans le cadre de son enseignement à I'EPHE, sur la construction rituelle de la catégorie de l'espace dans l'aire culturelle voltaíque. (Cartry 1975 : 79.92).

${ }^{8}$ Alain Supiot rappelle que le vocabulaire juridique reservart jusqu'à une date récente la notion d'espace « aux parties du monde qui, n'ayant pas de limites discern. ables et étant impropres à la vie humaıne, ne peuvent être dura. blement occupées ". comme les mers et les cieux. Par oppposition, la Terre «n'était pas appréhendée par le Droit comme un espace abstrait, mais comme un entrelacs » de lieux concrets et délimités : territoires, domaines, régions, pays, zones, sites. Cette précision de la langue juridique est d'autant plus précieuse qu'elle repose sur une distinction comparable à celle que le rite construit entre la brousse et le village. (Supiot, 2008 : 151). 
différenciés, l'évanouissement des limites, tel que cela se produit la nuit sans lune ou à midi, sous la lumière crue du soleil au zénith. Cette invisibilité de la brousse aux regards des vivants du village se donne à entendre dans l'association réitérée de la couleur noire aux " choses de la brousse ", mais nombre de faits suggèrent que les hommes sont vus, sinon regardés, depuis cette scène invisible (Cartry 1975 : 87). Une troisième propriété, liée à la deuxième, est l'action spécifique qu'exerce ce milieu sur le corps propre des chasseurs qui, à y rester trop longtemps, se trouve comme "vidé », " pompé ", " aplati ». Les contours du corps s'estompant, la personne risque de "s'évanouir ", au sens propre de "perdre connaissance ", comme au sens figuré de "disparaître " en finissant par se confondre totalement avec le milieu où elle se trouve (Cartry 1979 : 269).

Une fois connu cet élément du cadre général, essentiel à la compréhension de l'analyse qui va suivre, le texte aborde les faits qui se tiennent au point de départ de la réflexion, à savoir un ensemble de rites qui ne se laissent pas tous épingler comme rites de passages, et qui ont en commun de faire explicitement référence à la brousse alors même que leur raison d'être est de traiter une question à propos du corps et de ses limites. Pour l'essentiel, les rites retenus pour la démonstration sont des rites qui entourent la naissance et des rites concernant les jumeaux. On ne peut restituer ici la richesse des matériaux sollicités, et encore moins toute la subtilité de l'analyse qui tente de suivre la logique " en spirale " du rituel. Mais on s'attardera sur ce qui, selon nous, est à la fois au point de départ de la réflexion qui a conduit Michel Cartry à construire la notion d'espace corps, et au fondement de tous les développements qu'il lui donnera ultérieurement.

$\mathrm{Au}$ point de départ, se tient l'observation décisive selon laquelle, pour les Gourmantché, ce qui surgit à la naissance, ce n'est pas un corps, mais c'est un organisme double, formé de l'enfant et de son placenta. "On naît deux " énoncent-ils explicitement, et tout l'enjeu des gestes rituels effectués dans le temps qui suit l'accouchement sera de cerner et de décerner au petit d'homme un corps unique, 
tout en atténuant les effets potentiellement néfastes de cette mutilation. En plusieurs de ses écrits, Michel Cartry reviendra sur cette observation princeps, comme sur les spéculations gourmantché à propos du placenta, désigné comme le " compagnon " de l'enfant, son "deuxième ", son "plus soi que soi ", qui viennent éclairer de leur logique nombres de rituels gourmantchég . Il insistera, en particulier, sur le moment où la sage femme s'apprête à trancher le cordon, moment crucial dans le processus de l'accouchement qui " montre que ce dont l'enfant doit être séparé ce n'est pas du corps de sa mère mais d'une sorte d'organisme dont il est lui-même une partie " (Cartry 1987 : 203). À la différence de ce qui est en vigueur pour les accouchements médicalisés, le cordon n'est sectionné qu'à la toute dernière extrémité, une fois le placenta expulsé hors du ventre de la mère. Entre la sortie de l'enfant et l'expulsion du délivre, aucun mot ne sera prononcé, aucun geste ne sera effectué, la scène est comme figée, l'attente est palpable. Seule la délivrance remet les choses en mouvement, la sage femme sectionne le cordon, puis retourne l'enfant pour annoncer à la mère de quel sexe il est, tandis que les cris des autres femmes présentent dans la case de l'accouchement en avertissent ceux qui se tiennent au dehors. Ce que l'on attend, avant de trancher le lien, précisera Cartry en un texte ultérieur, c'est que chacune des personnes présentes dans la case de l'accouchement puisse "voir, non pas seulement l'enfant, mais l'organisme dans son entier ". "Tout se passe donc comme si ", ajoute-t-il, " après ce premier regard, le second regard posé sur l'enfant désormais séparé allait faire apparaître celui que dans nos cultures nous appelons un nouveau-né comme un être qui ne peut faire issue dans l'espace du village qu'en se séparant d'une partie de lui-même. Telle est l'énigme de la naissance. " (Cartry 1987 : 203).

Les gestes rituels gourmantché qui entourent la naissance et le traitement du placenta font surgir, d'une nouvelle façon, la question de la gémellité. Cette fois, "l'image du corps " des corps dédoublés des jumeaux n'est plus sollicitée comme forme structurante du temps et de l'espace vécu (mythe dogon), mais elle donne forme et contenu au rap-

\footnotetext{
9 Formulée en des termes proches en nombre de populations ouest africaines, cette théorie placentaire est, chez les Gourmantché, particulièrement développée au travers des rites, et non, comme dans les popula tions mandés issues de I'ancien Empire du Mali, dans des mythes cosmogoniques. En plusieurs de ses textes, Michel Cartry reviendra sur cette logıque placentaire, découvrant de nouveaux pans dans des rites dont l'objet immédiat n'est pas la naissance (excision, initiation masculine, fabrication d'un nouveau chef).
} 
port du nouveau-né à son placenta. Toute naissance repose l'énigme de cet organisme né double, mais qui ne peut vivre qu'unique. La question de ne pas retrancher l'organe placentaire ne se pose pas, mais les gestes rituels qui visent à préserver le délivre trahissent une forme d'inquiétude quant aux conséquences de cette mutilation sur le corps de l'enfant, dans le mouvement même où ils cherchent à en atténuer les effets. Les montages de représentation gourmantché autour du traitement du délivre laissent entendre que du sort du placenta dépend la stabilité future de l'enfant : un placenta égaré (il peut avoir été emporté par un animal ou, dans le contexte moderne, jeté par le personnel de la maternité avec d'autres déchets organiques) vouerait l'enfant à une vie d'errance et de la folie. En d'autres populations voltaïques, où l'on retrouve le même souci rituel d'une forme de préservation de l'organe placentaire, les discours insistent sur le lien qui perdure, par-delà la séparation d'avec l'enfant, entre les deux parties de ce qui a été un organisme unique pendant les neuf mois de la vie intra-utérine. Toute personne dont le placenta, soigneusement enterré dans un coin de sa demeure de naissance, viendrait à subir la chaleur d'un feu ou à être recouvert de sable, en ressentirait aussitôt les effets en son corps, elle aurait de la fièvre ou les yeux chassieux (Liberski-Bagnoud 2002). Le geste de la sage femme enterrant le placenta - il est placé dans l'eau d'une poterie qui est elle-même enfouie dans la terre à l'entrée de la case de la mère -, en même temps qu'il conjure ce risque, rappelle "que l'on ne peut rester deux, sauf sous la forme d'une identité dans la différence, le "deuxième " auquel on reste lié n'étant pas destiné à vivre de la vie des vivants "(Cartry $1987:$ 203).

Ainsi donc, toute naissance pose d'emblée la question des limites du corps propre, question à la fois liminaire et décisive, puisque c'est de sa résolution que va dépendre la "discernabilité " du corps comme tel. Ce sont, en effet, les gestes rituels de la sage femme et la formule conventionnelle qu'elle prononce qui vont, sur l'organisme qui a surgi dans la case de l'accouchement, cerner le corps du né-unique et le décerner comme corps sexué. À cet égard, 
la naissance de jumeaux soulève un redoutable problème, puisque une forme d'indiscernabilité va persister au-delà de la mutilation placentaire. C'est le paradoxe de la réalité gémellaire : issus d'une seule parturition et substantiellement identiques, les jumeaux n'en sont pas moins empiriquement deux ; et quoi que physiquement deux, il n'y a pour eux dans la structure généalogique de la famille qu'une seule place distincte ${ }^{10}$. Un montage complexe de représentations permet aux Gourmantché de reformuler cette nouvelle énigme, enchâssée dans celle de la naissance, en termes de proximité ontologique des jumeaux humains avec l'espace de brousse. Construite en miroir du territoire villageois, la brousse apparaît comme le lieu de projection imaginaire privilégié des bizarreries corporelles. Toutes sortes de créatures imaginaires la peuplent ${ }^{11}$, et, en particulier, une catégorie de petits êtres gémellaires chevelus appelés pola, dont le schème corporel comme l'habitus sont le symétrique inverse de ceux des humains. Ces petites créatures, qui ont pour caractéristique d'avoir conservé leur enveloppe placentaire dont elles se servent comme d'une cape d'invisibilité, sont la représentation fantasmatique de ce que pourrait être la vie des "non séparés ", doublement non séparé de leur organe placentaire et de leur jumeau : soudés deux à deux, parcourant la brousse en tous sens, sautillant, ne laissant aucune trace sur le sol, les pola n'ont aucune stabilité, aucun ancrage territorial. C'est avec ces petites créatures de brousse que les jumeaux humains sont, pour les villageois, dans une inquiétante familiarité. À leur naissance, des rites sont effectués afin de tester leur qualité d'humain et s'assurer qu'il ne s'agit pas d'êtres de brousse venus s'introduire par effraction dans le territoire villageois. Mais, malgré cela, toute leur vie durant, ils seront suspectés de conserver des liens étroits avec leurs doubles inversés de brousse.

On saisit la logique à l'œuvre : c'est en raison même de la forme d'indiscernabilité qui caractérise les corps des jumeaux que ceux ci sont symboliquement assignés à un espace aux contours mouvants et flous, qui a pour propriété de provoquer l'indistinction des corps. Cette parenté

\author{
${ }^{10}$ Ce « paradoxe \\ des jumeaux », \\ les anthropologues \\ Isaac Shapera (1930) et \\ Victor W. Turner (1969) \\ en avaient repéré les \\ termes dans les rituels \\ et les discours de \\ sociétés issues d'aires \\ culturelles différentes \\ (Khoisan d'Afrique du \\ Sud et Ndembu de \\ Zambie). \\ ${ }^{11}$ Ces créatures sont \\ toutes, peu ou prou, \\ des images déformées \\ du corps humain dont \\ certaines parais. \\ sent spécifiquement \\ renvoyer aux naissances \\ tératologiques : nain à \\ grosse tête, minuscules \\ êtres à jamais enfermés \\ dans leur sac placen. \\ taire (à l'image des \\ fœtus morts in utero), \\ créatures qui n'ont \\ qu'une moitié de corps, \\ avec un seul bras et \\ une seule jambe. \\ (Cartry 1975, 1979, \\ 1987).
}


12 S'annoncer au seuil de la case où le tout petit repose afin de ne pas le surprendre en train de converser avec les petites créatures de brousse, confec. tionner un plat de pâte de mil pour l'offrir " au compagnon de brousse " de l'étranger, donner une légère tape sur la tête de l'enfant qui persiste à marcher à quatre pattes afin de "lui fermer les yeux pour la brousse $\gg . .$. étroite des jumeaux humains avec la brousse relance, en retour, l'interrogation sur la nature des liens que l'enfant né unique conserve avec l'espace d'où il vient. D'autres moments du rituel, d'autres gestes quotidiens ${ }^{12}$ donnent en effet à comprendre que l'infans reste attaché à l'espace de brousse tant qu'il n'a pas acquis le langage des humains et la station debout, deux caractéristiques des petits d'homme. Son babil comme sa marche à quatre pattes sont les expressions visibles de cet attachement à la brousse, qui perdure bien au-delà de la mutilation placentaire. Lorsqu'il est né, des précautions ont été prises autour de la case de l'accouchement pour maintenir à distance les petits pola de brousse arrivés en masse, comme si l'arrivé d'un nouveau né avait induit un effacement des limites entre brousse et village tel que ce dernier avait été totalement envahi par la brousse et qu'il s'agissait de recréer autour de la case de l'accouchement une enclave de terre villageoise. La séparation d'avec l'organe placentaire opère un premier et indispensable détachement d'avec la brousse, un couteau placé pendant les premières semaines entre la peau de la mère et son nouveau né porté au dos en réalise un autre. D'autres gestes de la mère accomplis au cours du développement de l'enfant trahissent le même souci, qu'il s'agisse de faire cesser le babil de l'enfant avec les êtres de brousse, ou de lui "fermer les yeux pour la brousse " lorsqu'il tarde à adopter la marche debout. Pour les jumeaux, des rites spécifiques accomplis au moment du mariage réitèrent leur rapport singulier à la brousse, afin de tenter de les inscrire durablement dans le territoire du village.

L'existence de ces gestes et de ces rites en témoigne, le problème des limites du corps ne trouve pas sa résolution en seule fois, plusieurs moments clefs du développement et du cycle de vie d'une personne le font ressurgir, sous des termes à chaque fois différents, mais néanmoins reconnaissables en ce qu'ils mettent en jeu une structuration dynamique entre l'espace et le territoire, la brousse et le village. La référence à l'espace n'est pas ici simple affaire de code que manipulerait la pensée rituelle pour signifier un changement d'état social (telle la catégorie des " rites 
de passage " proposée par Van Gennep pour ranger toutes sortes de rites qui mobilisent des lieux - seuil, gué, porte, faille - dans le temps même où ils sont censés traiter une modification dans le statut d'une personne). Jumeaux, nouveau-né, infans, ne sont pas seulement symboliquement placés sous le signe de la brousse, et il ne s'agit pas non plus seulement de les en "détacher ", il faut aussi qu'ils s'inscrivent dans le territoire villageois, en intégrant les limites de ce dernier à celles de leur corps propre. On ne manquera pas de souligner la référence implicite qui est ici faite à la notion d'image du corps. Ainsi qu'il a été déjà dit, l'image du corps engage nécessairement une structuration spatiale dynamique, puisqu'elle suppose la différenciation entre un espace corporel et un espace extérieur, une acceptation des limites du corps et une compréhension de la relation des parties à la totalité. À l'instar des réflexions rapportées précédemment à propos des Dogon, tout se passe comme si, chez les Gourmantché, cette "image du corps " était prise en charge par la collectivité villageoise qui l'imposait par toute une série de rites à chacun de ses membres, dans le mouvement même où leur est transmis une certaine dialectique spatiale entre un Ailleurs de brousse d'où viennent les enfants et un territoire du village où ils auront à s'inscrire comme humains.

C'est très précisément cette dialectique que l'auteur cherche à saisir à partir de cette notion "d'espace-corps" qui vient là, pour la première fois, sous sa plume. Une lecture distraite pourrait faire croire, tout d'abord, que l'expression ne renvoie qu'à une manière de concevoir le village sur le modèle d'un grand corps orienté. On apprend, en effet, qu'en gulmancéma, langue des Gourmantché, il n'y a pas d'autre terme pour désigner ce que nous appelons un " village " que celui de " corps terre unique " - tin'gban'yendo, littéralement " terre peau un " (notons qu'il n'y a en cette langue pas d'autre façon de désigner le corps vivant qu'en nommant ce qui fait sa limite empirique, sa frontière physique, la peau). Dans ce corps du village, les grandes divisions spatiales que l'on appelle communément des " quartiers " sont les organes, la bouche (à l'ouest), le ventre (au centre), 
l'anus (à l'est). Autrement dit, l'espace-corps serait la façon endogène de dire le " territoire "dont l'inévitable résonnance politique tend à masquer le sens qu'il peut avoir en anthropologie, à savoir celui d'un « espace qui a été balisé de telle sorte que pouvant le reconnaître sien, on peut s'y déplacer sans danger " (Cartry 1979 : 278). La suite du texte empêche toutefois de s'en tenir à cette équivalence simple, enfermée dans une ethnographie particulière. Il est en effet précisé que c'est « l'appropriation d'un territoire (qui) rend nécessaire, au-delà de l'apparente limite d'un corps que représente la peau, le marquage d'un espace-corps " (ibidem). La notion d'espace-corps n'a pas pour référent une représentation mythique figée de l'espace vécu d'une communauté villageoise donnée, mais elle nous renvoie à des pratiques, ici ritualisées, et sa question se pose à tout groupe humain. Le territoire, dans son acception anthropologique, n'est pas qu'une histoire de conquête politique et d'aménagements économiques et sociaux, mais il émerge de la transformation symbolique et imaginaire d'un espace quelconque en un lieu aménagé de manière telle que le corps humain puisse y perdurer dans sa forme, sa stabilité, son système d'axe, ses limites. Ici espace collectif, symboliquement façonné à l'image d'un corps, avec ses orifices, ses six directions et ses trois axes (haut/bas, devant/derrière, gauche/droite), l'espace-corps est par définition un prolongement du corps propre, au delà de ses limites naturelles. La singularité gourmantché, et, au-delà, celles des populations voltaïques et mandé, est d'avoir fait de cet espace-corps, à la fois contenant et limite, également une surface d'inscription où, par le biais du traitement de l'organe placentaire, le rattachement à une chaîne d'ancêtres est symboliquement assuré (Cartry 1975 : 90). A contrario, l'espace de brousse se définit précisément de n'avoir pas les propriétés d'un corps. Sans limites discernables, il ne peut s'offrir ni comme contenant ni comme surface d'inscription, et ne peut donc être durablement occupé par les humains. On retiendra encore ceci : les productions fantasmatiques des Gourmantché à propos des petites créatures pola, ces " vivants non encore nés, à jamais enfermés dans leur sac placentaire et à jamais 
couplé à leur jumeau "(ibidem), disent en creux que c'est au prix d'une double perte (celle de son deuxième placentaire et celle de son milieu originel) que le petit d'homme intègre l'espace-corps que lui offre la communauté villageoise. Néanmoins, l'existence même d'une fantasmagorie mettant en scène des créatures qui auraient choisi de ne pas subir cette mutilation, et, par-là même, de n'avoir pour seul " espace-corps » que celui que leur offre l'enveloppe placentaire, cela nous indique clairement, souligne Michel Cartry, que, pour les Gourmantché, cette perte ne va pas de soi.

C'est donc, tout d'abord, pour chercher à conceptualiser ces montages de représentations qui construisent le rapport des corps au village différemment de celui des pola à la brousse, que la notion d'espace-corps a été forgée. L'approfondissement de ses recherches sur la nature et la fonction du rituel, tout au long de son enseignement, vont lui permettre d'en préciser les contours. Conduisant des analyses approfondies de ces "lieux ", éphémères ou durables, que fait émerger le rituel (lieux de la maison, camp de circoncision, lieu de la retraite initiatique du chef, table divinatoire, etc.), il montrera qu'en nombre de cas, ces espèces d'espace sont dotés par le rite de propriétés similaires aux propriétés d'un corps. On ne peut ici évoquer toutes ces recherches, tant leur diversité est grande, mais il nous faut mentionner celle sur un " camp de circoncision ", à laquelle il consacra plusieurs années d'enseignement, et un compte rendu substantiel en 1991 oủ l'on trouve l'une des rares occurrences écrites de la notion d'espace-corps. Étudiant en détail les rites qui président à l'installation de l'enclos initiatique, l'ethnologue va s'intéresser tout particulièrement à ces lieux de l'enclos qu'il définit comme "lieux à haute tension " que sont les ouvertures, les lieux de dépôt des plats de nourriture, le trou de recueillement des urines des circoncis, les places qu'ils occupent pendant les chants, etc. Découvrant l'existence d'un parallélisme étroit entre le traitement rituel de ces lieux de l'enclos et celui des "lieux du corps " des initiants, il démontrera que l'enclos de circoncision présente les propriétés d'un espace-corps, qu'il définit alors comme un " organisme vivant " (Cartry 
1991 : 57) dont les lieux sont appréhendés dans le rite comme autant de "lieux du corps "(Cartry 1989 : 90). De cette lecture, on retiendra l'interdépendance de l' "image spatiale du corps " (ou espace $d u$ corps) et de l'espace-corps : l'investissement par le rite des lieux construits de l'enclos initiatique permet d'opérer une transformation de l'image du corps des initiants qui, entrés dans le camp de circoncision comme créatures prématurées, enfantées par les Masques, en sortiront comme des adultes sexués, membres à part entière de la société gourmantché (Cartry 1991). De cette interdépendance - qui n'est pas confusion entre l'espace $d u$ corps et l'espace-corps qui, toujours, renvoie nécessairement à un espace extérieur au corps propre - nombre d'autres analyses de rites conduites par Michel Cartry en apporteront la preuve. On ne peut ici que mentionner, sans les commenter, ses enquêtes sur la notion de souillure du meurtre et celles sur le deuil, où il construit l'hypothèse selon laquelle les irruptions organiques au sein du village (mort, accouchement, meurtre, circoncision), en plongeant les patients du rite (endeuillé, accouchée, meurtrier, circoncis) dans un état d'indistinction (ni vivant ni mort, ni homme ni femme), sont toujours appréhendées comme venant fragiliser les limites d'un espace-corps du village que les rites auront à réaffirmer avant de chercher à sortir les patients de leur état.

Mais c'est dans les derniers développements de la recherche de longue haleine qu'il a conduite sur la géomancie qu'il dévoilera la place cardinale qu'il entend donner à l'espace-corps dans la théorie du rituel qu'il n'a eu de cesse de construire pas à pas, au fil d'analyses exigeantes et méticuleuses des pratiques rituelles d'une société donnée. Dans l'un de ses derniers articles, consacré à l'écriture divinatoire, il construit l'hypothèse selon laquelle l'espace d'écriture qui surgit sous la main du géomancien est bien autre chose qu'une simple "feuille d'inscription ", c'est, affirmet-il, une sorte "d'espace-corps " (Cartry, 2005 : 416). Ce qu'a fait apparaître la main du devin, alors qu'il trace devant lui, très rapidement, les quatre premières séries de points d'où il tirera, par calcul successif, les différentes figures géo- 
mantiques, c'est en effet un espace orienté dont les noms indexent des parties du corps : la partie la plus proche de lui, le géomancien l'appellera "ventre du sable ", la plus éloignée, " dos du sable ", celle sur sa gauche "le fondement du sable ", ou son " début ", celle sur sa droite, "la bouche du sable ", ou encore "la terminaison du sable ". Nombre de géomanciens insistent sur le fait que pour comprendre la façon dont s'ordonnent les trajets de la main du devin, il faut prendre en compte ce schème de représentation d'un espace-corps ainsi orienté. Selon leur dire, tracer des points d'une ligne qui va de gauche vers la droite, c'est suivre un trajet partant d'un " lieu du corps " de la Terre en son commencement jusqu'à ce point de terminaison que constitue l'orifice de la "bouche du sable " où, après un décompte par deux des points tracés, il recueillera le reste, sorte de balbutiements dont l'addition successive permettra de former une première figure. Ces trajets de gauche à droite qu'opère la main traçante du devin, tout en faisant émerger l'espace-corps de la terre, "suivent comme les trébuchements d'une parole de la terre à divers moments de son cheminement " (idem). L'espace-corps acquiert la dimension d'un organisme vivant à la surface duquel vient s'écrire la parole de la Terre. Sur les différentes phases suivies par le devin pour extraire cette parole de "la bouche du sable ", Michel Cartry livrera une analyse serrée. Ce qu'il faut ici retenir, c'est le parallèle qu'établit le travail du rite entre l'extraction de cette parole oraculaire et le désir de délier le corps propre du consultant des effets d'une parole prononcée en un temps prénatal, mauvais nœuds et collets où prennent origine les problèmes de maladie, de procréation, ou d'Avoir impossible.

Cette "fiction rituelle», selon le terme que l'auteur réserve à ces productions symboliques que met en œuvre le rituel par des procédés d'agencement et de mises en scène qui lui sont propres est, pour ainsi dire, l'analogon de l'agir rituel. Michel Cartry aura prêté une attention toute particulière au fait que le traitement des corps et de l'espace est une caractéristique générale du travail du rite. Il n'aura eu de cesse de montrer qu'il n'existait guère de rites où les gestes, 
les objets, les mots ne soient mobilisés pour traiter un problème d'espace (discrimination des lieux, jeux d'opposition sur l'extérieur/l'intérieur, le haut/le bas, le devant/le derrière, redéfinition des limites, etc.). De même, l'implication des corps dans le rite est récurrente et centrale, "du " corps ou des morceaux de corps y sont l'objet d'une série d'opérations, alors même que les rites en question n'ont pas pour fonction explicite de guérir ou de modifier l'aspect des corps. Que l'espace et les corps, ne soient pas simplement le cadre ou l'un des éléments du rite, mais les points d'application sur lesquels s'exerce le travail du rite pour résoudre certaines antinomies, c'est bien l'une des énigmes majeures que pose l'agir rituel, comme l'avait déjà vigoureusement indiqué en son temps Marcel Mauss. En ayant eu très tôt le souci de saisir l'étroite articulation qui existait entre rite et divination, entre l'agir rituel et la parole oraculaire, Michel Cartry a ouvert un chemin qui pourrait conduire à la résolution de cette énigme. Dans le projet d'ouvrage déjà cité, on trouve dans une note liminaire une indication sur la thèse qu'il comptait développer en ce travail.

"La divination et le rituel ", peut-on y lire, "sont des actes qui se déploient dans un espace qui n'est ni celui de la pensée ni celui de l'action, mais qui se présente comme une sorte d'espace-corps oủ les acteurs doivent à chaque fois se réinscrire pour penser et habiter leur monde, le prêtre et le devin ayant en charge de traiter ce qui s'est mal noué dans le rapport du corps au langage. "

Cette précieuse note qui livre la pointe de la réflexion à laquelle l'a conduit son étude du rituel et de la divination dans les sociétés subsahariennes indique la place princeps qu'il entendait donner au concept d'espace-corps dans une théorie du rite. Résolument neuve, cette théorie recèle l'intuition profonde, dont il a pu faire part dans son enseignement, que dans le rite, du corps est toujours pris, le corps de l'agent du rite mais aussi un corps-monde qui rappelle le monde d'avant le langage, où chaque son est bruit du

${ }^{13}$ Conférence de l'EPHE, décembre 1998. monde, chaque odeur, odeur du monde. Ce monde du narcissisme primaire, forclos par la science, mis en souffrance dans nos sociétés technoscientifiques, disait-il, le rite n'a de cesse de le mettre en scène et de le "travailler ", afin de chercher à desserrer autant que possible la « corde " du destin ${ }^{13}$. 


\section{Références bibliographiques}

Adler, A. \& Cartry, M.

1971 "La transgression et sa dérision ", L'Homme, XI, 3, pp. 5-63.

Cartry, $M$.

1979 "Du village à la brousse ou le retour de la question. À propos des Gourmantché du Gobnangou (Haute-Volta)", in M. Izard et P. Smith (dir.), La Fonction symbolique. Essais d'anthropologie, Paris, Gallimard (Bibliothèque des sciences humaines), pp. 265-288.

1987 "Le suaire du chef ", in M. Cartry (dir.), Sous le masque de l'animal. Essais sur le sacrifice en Afrique noire, Paris, PUF (Bibliothèque de l'École des Hautes Études. Sciences religieuses, vol. LXXXVIII), pp. 131-231.

2005 "Eine Orakelschrift / Une écriture divinatoire ", in R. Baur (ed.), Das gesetz und seine visuellen folgen / La Loi et ses conséquences visuelles, Leiptzig, Lars Müller Publishers, pp. 402-429.

Résumé des conférences ct travaux de l'EPHE (Section des sciences religieuses)

1973-74 "Recherche portant sur les croyances, les mythes et les rites liés aux jumeaux ", t. 82, pp. 17-38.

1974-75 "Analyse de catégories religieuses liées à l'idée de génération et de procréation ", t. 83, pp. 35-39.

1975-76 "Recherche sur la catégorie de l'espace " dans l'aire voltaïque, t. 84, pp. 79-94.

1984-85 "Rituels gourmantché [...] de la maison", t. 93, pp. 73-75.

1989-90 et 1990-91, "Rites initiatiques de circoncision chez les Gourmantché du Burkina Faso " (l'enclos initiatique), t. 98 et t. 99, pp. 86-93 et 47-53.

1991-92 "Espaces initiatiques [...] ; recherches comparatives " (Moose, Kurumba, Songhay-Djerma, sociétés bantoues, t. 100, pp. 57-61.

Archives (déposées à l'Institut d'Études Avancées de Nantes).

1973 Titres et Travaux. Programme d'enseignement. Dossier de candidature au poste de Directeur d'études à l'EPHE. Cote : 5DMC1

Notes en vue de la préparation d'un ouvrage sur la divination (intitulé ici "bouche de la terre ". Cote : 3DMC64

Dolto, F.

1992 L'Image inconsciente du corps, Paris, Editions du Seuil.

Liberski-Bagnoud, D.

2002 Les Dieux du territoire. Penser autrement la généalogie, Paris, Éditions du CNRS.

Merleau-Ponty, M.

1945 Phénoménologie de la perception, Paris, Gallimard (Collection Tel), Irééd. 1976]. 
Nishitani, O.

2013 "Le japonais ", in P. Legendre (dir.), Le Tour du monde des concepts, Paris, Editions Fayard, "Poids et Mesures du Monde".

Pankow, G.

1962 "Das Körperbild bei einem Hopi-Indianer ", Zeitschrift für Psycho-somatische Medizin 8, $\mathrm{n}^{\circ}$ 3, pp. 223-226. [Thèse reprise dans] L.'Homme et son espace vécu, $2^{ }$éd. augm. et révisée, 1986, Paris, Aubier, pp. 19-26.

1977 Structure familiale et psychose, Paris, Editions Aubier Montaigne

Poupart, F.

2014 "Hommage à Gisela Pankow », Psychothérapie, 2014/1, vol. 34.

Schapera, I.

1927 "Customs relating to Twins in South Africa ", Journal of the African Society 26, pp. 117-137.

Supiot, A.

2008

"L'inscription territoriale des lois ", Esprit, novembre, pp. 151-170.

http://www.iea-nantes.fr/fichier/j_telechargement/13/telechargement_ fichier_fr_as.inscription.territoriale.des.lois.esprit_nov08.pdf

Turner, V. W.

1969 The Ritual process : Structure and Anti Structure, Londres, Routledge \& P. Kegan. 